\title{
Correspondence
}

Editor: Ian Pullen

Contents: Family therapy for schizophrenia/Better to be depressed in Australia .../... or not?/AIDS hypochondriasis/Journal contents page/Obstetric liaison/Calcium techniques/Neuroleptic malignant syndrome/NMS and thioxanthenes/Schizophrenia, season of birth and maternal age/Homosexual erotomania/Post-traumatic stress disorder/Neuronal misconceptions/Unreliable urine samples/ Munchausen's syndrome by proxy/Sorcery and psychiatry/Solvent abuse psychosis.

\section{Family therapy for schizophrenia}

SIR: Leff et al (Journal, January 1989, 154, 58-66) recommend establishing relatives' groups, in conjunction with family education and one or more initial family therapy sessions in the home, as the most cost-effective and efficient management of schizophrenic patients maintained on neuroleptic medication and living in contact with relatives having high expressed emotion (EE).

Before incorporating this recommendation into routine clinical practice, several questions need to be addressed. The results of the two-year follow-up were not entirely clearcut, since 2 of 12 patients in the experimental group committed suicide. Professor Leff $e t$ al do not report on the long-term follow-up of their first trial of intervention. This data seems essential before concluding that intensive social interventions are really cost-effective, given the considerable demand on professional resources.

Professor Leff $e t$ al refer to difficulty in accumulating sufficient families. Could they elaborate? Our own research on the course and outcome of first lifetime episode schizophrenia suggests that at this stage of the disorder, parents are not prepared to consider family therapy (independent of EE status), but are prepared to participate in relatives' groups in conjunction with family education and participation in planning rehabilitation (Waring et al, 1988). Our experience suggests a lack of efficiency in accumulating sufficient families for family therapy at this early stage of the disorder. An alternative explanation is that the process of engagement of these families requires more than "tact and patience", and perhaps skills that "ordinary" clinicians, not involved in high profile research projects, do not possess. We are aware of very few clinical psychiatrists who in their treatment of schizophrenia are able to combine educational, behavioural, structural, and systemic techniques of family therapy.

One other explanation of our "low efficiency" is that the high EE families studied by Professor Leff $e t$ $a l$ are unusually co-operative and compliant in participating in a family therapy package. A young person with a first episode of schizophrenia is fortunate to have a family who are considerate and tolerant. This attitude of concern appears to be independent of the EE status of the family or whether or not they are treated with social interventions (Johnstone et al, 1986; McCreadie et al, 1987).

We would also appreciate a clarification of what exactly Professor Leff $e t$ al mean by "face to face" contact. Does it refer to a patient and a relative being in the home at the same time? Our experience suggests that few adolescent schizophrenics interact directly with relatives for five hours a day. Perhaps another term would be more helpful?

Finally, the programme recommended appears to have provided family therapy to only six families per year! Is it possible that the treatment effectiveness reflects an alteration of parental attitude from fear and hostility towards a child's perceived deviant behaviour to an attitude of compassion and sympathy for a child with a "broken brain"? If so, it is not surprising that, for the short term, a family can express tolerance towards a schizophrenic child.

We greatly admire the research of Professor Leff $e t$ al. Our question is whether, and how, to transfer family therapy approaches from 'select' research samples of patients and their compliant families to the vast majority of out-patient schizophrenics seen in day-to-day clinical practice.

\section{E. M. WARING R. MANCHANDA \\ C. CARver}

University Hospital

London

Ontario

Canada N6A 5A5. 


\section{References}

Johnstone, E. C., Crow, T. J., Johnson, A. L. et al (1986) The Northwick Park Study of First Episodes of Schizophrenia. British Journal of Psychiatry, 148, 115-120.

Scottish Schizophrenia Research Group (1987) The Scottish First Episode Schizophrenia Study. British Journal of Psychiatry, 150, 331-333.

Waring, E. M., Lefcoe, D. H., Carver, C. et al (1988) The course and outcome of early schizophrenia. Psychiatric Journal of the University of Ottawa, 13,194-197.

SIR: Dr Waring et al raise a number of questions about our attempts to help the families of schizophrenic patients. We have not followed up our two trials beyond two years. Although we recognise the importance of a longer perspective, it is difficult to maintain control over the interventions received beyond two years. For the same reason, it is rare for trials of maintenance drugs to exceed this period. Uncontrolled follow-ups are of doubtful value; however, we did maintain contact with a number of families for three years or more and did not learn of any further suicides. Moreover, there were no suicides in our second trial, which may reflect improved intervention techniques, or merely better luck. With regard to the demand on professional resources, this is maximal during the first year of intervention for most families. Beyond that, families appreciate having access to professional help from time to time, but in many cases a phone call or letter once a month or less is sufficient.

Our difficulty in accumulating sufficient families was not due to their reluctance to accept the help offered, but to our stringent entry criteria for the trials. We focused on the highest risk families; those in which patients were in high face-to-face contact (more that 35 hours per week in the same room as the relative) with high EE relatives. These constitute no more than 1 in 6 of all schizophrenic admissions. We recognize the limitation this imposes on generalising our findings to "the vast majority of outpatient schizophrenics seen in day-to-day clinical practice", and have never claimed that our recommendations extend to all patients living with families. Indeed, the study of the Scottish Schizophrenia Research Group (1987) reveals that for patients living with relatives in a rural community, the prognosis for schizophrenia is remarkably good, only $19 \%$ being readmitted in the course of a year.

Our recommendations apply, not to these fortunate individuals, but to the high-profile patients who are frequently readmitted despite receiving drug therapy. We are therefore less concerned with the first onset patients that Dr Waring et al are attempting to help, although we do attempt to engage their families if they are high EE, high contact. We have not experienced any insuperable obstacles to this, perhaps because we begin the process of engagement by offering knowledge about the illness, for which relatives are eager, and follow on by attempting "to help the family to help the patient". We never target the family as the object of "therapy". An additional factor which facilitates engagement is our readiness to make home visits to those families who are unwilling or unable to come to us.

It is our view that reduction in high-EE attitudes does represent an increase in tolerance and understanding for the sufferer. However, this is also accompanied by significant changes in the coping behaviour employed by relatives. We are in the process of studying these in more detail.

Finally, we share the concern of Dr Waring et al that our intervention techniques may be perceived as beyond the capability of ordinary clinical teams. This has impelled us to study whether the techniques can be learned and effectively applied by psychiatric nurses in a routine clinical service. We shall report on this research in the fullness of time.

MRC Social Psychiatry Unit
Institute of Psychiatry
De Crespigny Park
London SE5 8AF

JULIAN LEFF

RUTH BERKOWITZ Christine VaughN

\section{Better to be depressed in Australia ...}

SIR: Kiloh et al (Journal, December 1988, 153, 752757) describe better outcome after 18 years among New South Wales depressives than among our Maudsley patients (Lee \& Murray, 1988). They suggest that the poorer outcome in London may have been due to the mis-inclusion of schizophrenic and schizoaffective cases, and are inclined to dismiss our findings concerning the predictive power of the neurotic-psychotic continuum on similar grounds.

We have re-analysed our data after excluding all those patients who developed schizophrenia and schizoaffective disorder: $25 \%$ of the remainder still fell into the operationally defined poor-outcome group. We further examined whether the presence of delusion, hallucinations, or mood incongruent features during the index admission predicted poor outcome. None of these indicators of possible "misinclusion" were correlated with poor outcome. Finally, and most surprisingly, we found that the development of schizophrenia or schizoaffective 